\title{
Case Report: Dramatic Response to Crizotinib in a Patient With Non-Small Cell Lung Cancer Positive for a Novel ARL1-MET Fusion
}

\author{
Qing Ma, Lingping Kong and Diansheng Zhong* \\ Department of Oncology, Tianjin Medical University General Hospital, Tianjin, China
}

\section{OPEN ACCESS}

Edited by:

Nehad M. Ayoub,

Jordan University of Science and

Technology, Jordan

Reviewed by:

Geeta G. Sharma,

City of Hope National Medical Center,

United States

Charles Leduc,

University of Montreal Hospital Centre (CRCHUM), Canada

Kurtis Davies,

University of Colorado, United States

Puyuan Xing,

Chinese Academy of Medical

Sciences and Peking Union Medical

College, China

${ }^{*}$ Correspondence:

Diansheng Zhong

Dianshengzhong@163.com

Specialty section:

This article was submitted to

Pharmacology of Anti-Cancer Drugs,

a section of the journal

Frontiers in Oncology

Received: 29 October 2021

Accepted: 19 January 2022

Published: 14 February 2022

Citation:

Ma Q, Kong $L$ and Zhong D (2022) Case Report: Dramatic Response

to Crizotinib in a Patient With

Non-Small Cell Lung Cancer Positive

for a Novel ARL1-MET Fusion.

Front. Oncol. 12:804330.

doi: 10.3389/fonc.2022.804330
It is imperative to know the status of oncogenic drivers in patients with non-small cell lung cancer (NSCLC). Compared with ALK and ROS1 fusion, MET fusion is relatively rare in NSCLC. In this case, we report the case of a female patient with NSCLC positive for a novel ARL1-MET fusion. The patient achieved about a 5-month progression-free survival (PFS) after receiving crizotinib for unresectable right lung malignancies. To the best of our knowledge, this case provides the first clinical evidence that the novel ARL1-MET fusion might be an actionable mutation in NSCLC.

Keywords: non-small cell lung cancer, ARL1-MET fusion, crizotinib, case report, targeted therapy

\section{INTRODUCTION}

Non-small cell lung cancer (NSCLC) is a disease commonly caused by alterations in oncogenic drivers. MET fusion is relatively rare compared with $A L K$ and ROS1 fusion in NSCLC. To date, eight MET fusion partners have been reported for NSCLC: ATXN7L1, KIF5B, STARD3NL, SPECC1L, HLA-DRB1, UBE2H, SLC1A2, and PTPRZ1. MET fusion has been reported to be a therapeutic target in lung cancer (1-6).

Crizotinib is an orally bioavailable, adenosine triphosphate (ATP)-competitive, small-molecule inhibitor of the receptor tyrosine kinases (RTKs) c-MET [also known as hepatocyte growth factor receptor (HGF)], ALK, and ROS1. When the drug was initially developed, it was mainly used as a small-molecule $M E T$ inhibitor and has been proven effective in preclinical and phase I clinical trials (7). It can inhibit the proliferation and invasion of HGF-activated endothelial cells, and the antitumor effect is dose dependent, which is closely related to the inhibition of MET phosphorylation (8).

Herein, we reported the case of a 60 -year-old woman with NSCLC. This patient was found to have synchronous multiple primary lung cancer (SMPLC), which meant that the time since tumor discovery was less than 6 months. The patient underwent thoracoscopic wedge resection of the left upper lobe and dissection of the mediastinal lymph node. Histologically, postoperative pathology confirmed that the left lung tumor was adenocarcinoma, while the unresectable right lung tumors were confirmed to be adenosquamous carcinomas through bronchoscopic biopsy. A novel ADPribosylation factor, GTPase 1 (ARL1)-MET fusion, was detected in the right lung tumor tissues using next-generation sequencing (NGS). She obtained a 5-month progression-free survival (PFS) after receiving crizotinib for the unresectable right lung malignancies. 


\section{CASE DESCRIPTION}

In July 2018, a 60-year-old woman was admitted to our department with complaints of chest tightness and dizziness. She had no other accompanying symptoms such as cough, expectoration, or hemoptysis. She reported a 1-year history of diabetes and was receiving treatment with metformin and voglibose. Thoracic CT showed a subsolid nodule under the pleura of the upper left lobe, approximately $1.1 \mathrm{~cm}$ in diameter. The edge of the nodule was rough, indicating a malignant lesion. No metastasis to the head or bone was observed. Of note, thoracic CT showed no nodule in the right lung (Figure 1A). In August 2018, the patient underwent thoracoscopic wedge resection of the upper left lobe and mediastinal lymph node dissection. Postoperative pathology revealed that the tumor was an invasive adenocarcinoma without invasion of the pleura (Figure 1B). Additionally, lymph node 6 was negative. The patient was diagnosed with stage IA lung adenocarcinoma (pT1bN0M0, Figure 1B). Subsequently, she was followed up regularly, and no accompanying symptoms were observed. On December 6, 2018, thoracic CT showed a soft tissue density nodule in the right middle lobe and near the hilum, approximately $20 \mathrm{~mm}$ in diameter (Figure 1A). On January 25, 2019, thoracic CT revealed that the size of the soft tissue density nodule in the right middle lobe near the hilum had increased to $35 \times 29 \mathrm{~mm}$. Meanwhile, multiple enlarged lymph nodules in the right hilum and mediastinum were observed, indicating metastatic lesions. On February 28, 2019, PET/CT showed multiple enlarged lymph nodes in the right neck and chest, as well as a soft tissue mass in the right hilar region. The former were considered to be metastases and the latter a malignant lesion. Subsequently, she underwent a bronchoscopic biopsy. Hematoxylin and eosin (H\&E) and immunohistochemical staining of tumor tissues taken from the right lung showed characteristics of adenosquamous carcinoma (Figure 1B). Of note, the presence of TTF-1 staining in the p40-positive cells may be due to a less-specific anti-TTF-1 primary antibody (SPT24). The tumor in the right lung was confirmed to be stage IVA NSCLC with squamous and glandular differentiation with a favored diagnosis of adenosquamous carcinoma according to the WHO Classification of Tumors, 5th Edition (pT4N3M1a). To seek personalized treatment strategies, formalin-fixed paraffinembedded (FFPE) sections of tumor tissues from the right lung of the patient were subjected to NGS using a 68-gene panel associated with the pathogenesis and targeted therapy of lung cancer (Burning Rock Biotech, Guangzhou, China) on March 6, 2019. The average sequencing depth is $1,887 \times$. NGS revealed a novel ARL1-MET rearrangement (fusion) in the tumor tissue sample from the right lung. The breakpoints occurred within intron 1 of $A R L 1$ and within exon 14 of MET. Therefore, the ARL1-MET fusion protein in the patient was supposed to retain the completed tyrosine kinase domain of MET, which conferred potential oncogenic activity (Figurea 2A, B; Table 1).

To clarify the mutation profile of adenocarcinoma in the left lung, FFPE sections of tumor tissues resected from the left lung of the patient were subjected to NGS using a 500-gene panel (CSO 500, ChosenMed Technology (Beijing) Co. Ltd., Beijing,

A
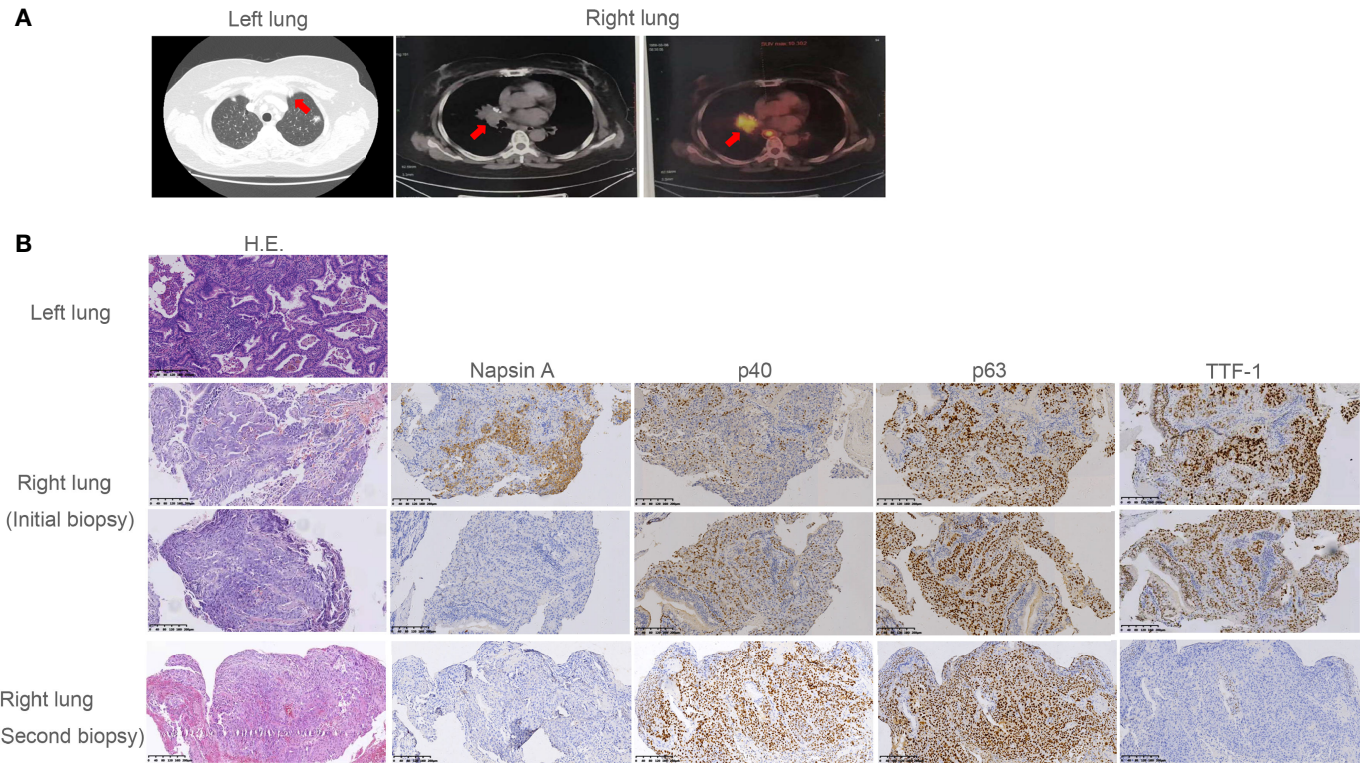

FIGURE 1 | Radiographic imaging at diagnosis and pathological findings. (A) The subsolid nodule under the pleura of the upper left lobe and the soft tissue density nodule in the right middle lobe and near the hilum revealed by computed tomography (CT) or positron emission tomography (PET). (B) Pathological examination revealed the subsolid nodule under the pleura of the upper left lobe was adenocarcinoma. Pathology showed the initial and the second biopsy of soft tissue density nodule in the right lung were adenosquamous carcinoma and squamous carcinoma, respectively. Interpretation of immunohistochemistry (IHC) performed on the initial biopsy was challenging as most tumor cells were TTF-1 positive suggesting adenocarcinoma. However, there was clear and diffuse positivity for p40 in some cells with corresponding lack of Napsin A. This suggested both glandular and squamous differentiation. In the second biopsy, p40 was diffuse with complete lack of adenocarcinoma markers TTF-1 and Napsin A, consistent with squamous cell carcinoma. 
China) on September 28, 2021. The average sequencing depth was 347.4×. The results of NGS analysis are shown in Table 2. Using a combination of pathological analysis and mutation profile, SMPLC was diagnosed in the patient (Figures 1A, B; Tables 1, 2).

The patient was started on off-label crizotinib treatment (p.o., b.i.d.). Three weeks after treatment with crizotinib, cough and dyspnea were remarkably relieved. In June 2019, thoracic CT showed that the size of the nodule shadow in the right middle lobe was reduced to approximately $22 \mathrm{~mm} \times 14 \mathrm{~mm}$, and multiple lymph node shadows in the right pulmonary hilum and mediastinum were also greatly reduced. She achieved a partial response (PR) defined by RECIST1.1 (Figure 3). However, by August 22, 2019, the patient's disease had progressed rapidly, and there was no improvement even after two cycles of paclitaxel plus carboplatin-based chemotherapy. A second biopsy was performed on December 24, 2019 to clarify the underlying mechanism of resistance to crizotinib. Pathology revealed that the adenosquamous carcinoma in the right lung had transformed into squamous carcinoma (Figure 1B). FFPE sections of tumor tissues were subjected to NGS through an 825gene panel (Genetron Health (Beijing) Co. Ltd., Beijing, China) with an average sequencing depth of $1,609.93 \times$. The results revealed that the original ARL1-MET fusion disappeared but
ERBB2 insertion (NM_004448: exon 20: c.2313_2324dup: p.Y772_A775dup) appeared (Table 3). The patient declined any inhibitors against ERBB2 insertion for personal reasons. She died 6 months later. The case timeline is shown in Figure 4.

\section{DISCUSSION}

In this case, a previously undescribed fusion of ARL1 exon 1 (NM_001177) to part of MET exon 14 (NM_000245) was detected in a patient with NSCLC and SMPLC using NGS.

$A R L 1$ is located at $12 \mathrm{q} 23.2$, encoding a protein belonging to the ADP-ribosylation factor-like (ARL) family of proteins. ARL1 is the first identified member of the large ARL family, which serves as an important regulator of the Golgi complex structure and is involved in several cellular processes, such as the endosomal trans-Golgi network, secretory trafficking, and innate immunity (9). ARL1 has six exons.

The novel ARL1-MET fusion protein was predicted to contain exon 1 of $A R L 1$, consisting of only one full codon. Thus, the fusion would essentially be a truncated version of $M E T$, which preserved the complete tyrosine kinase domain of $M E T$ (Figure 2B). Histologically, the patient's SMPLC was diagnosed as NSCLC. She underwent surgery for early

A

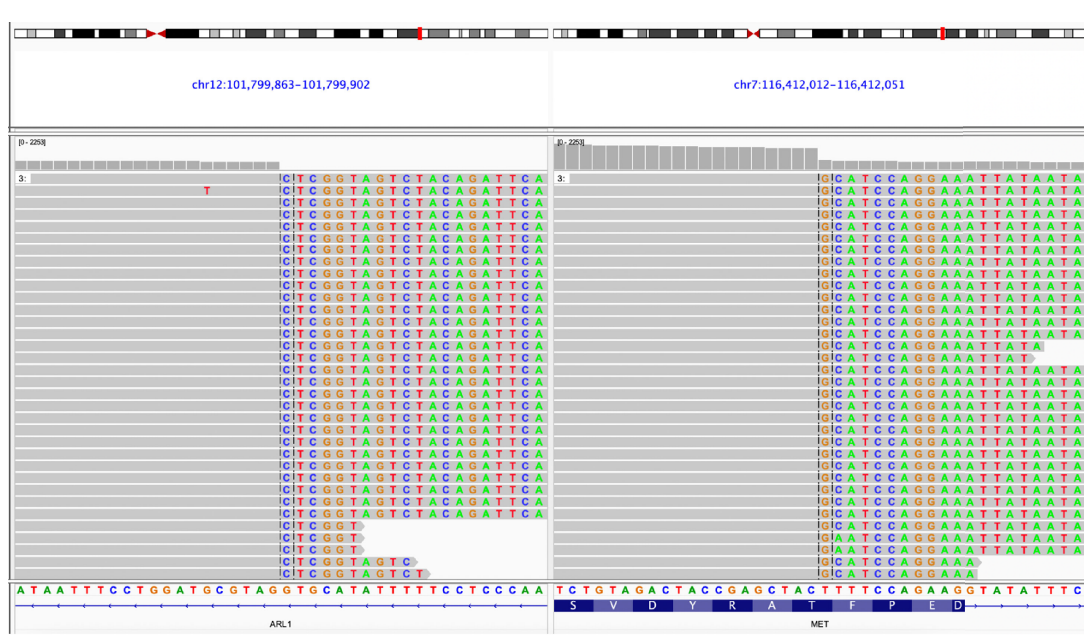

B

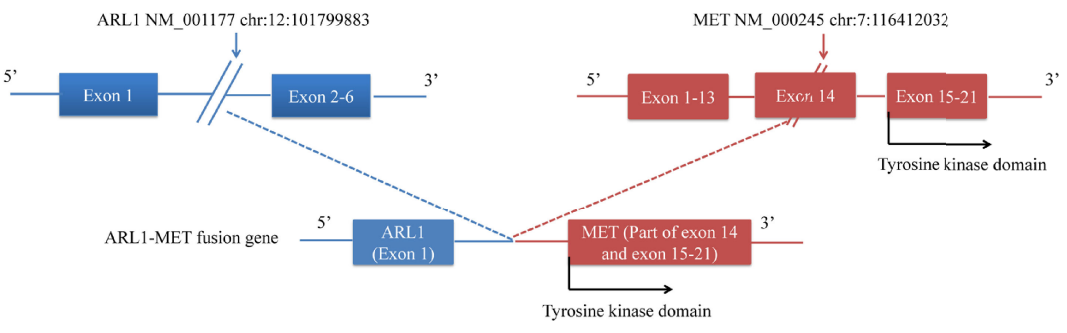

FIGURE 2 | ARL1-MET fusion was detected in the patient. (A) Sequencing reads of the ARL1-MET fusion revealed by the Integrative Genomics Viewer (IGV). (B) Illustration of ARL1-MET fusion. 
TABLE 1 | Mutation profile of right lung tumor in the patient before treatment with crizotinib.

\begin{tabular}{|c|c|c|c|c|c|}
\hline Gene & Transcript & Exon & Nucleotide change & Alteration & Mutant allele frequency \\
\hline \multirow[t]{2}{*}{ ARL1-MET fusion } & ARL1:NM_001177 & 1 & ARL1 (Exon1)-MET (Exon14) & & $74.27 \%$ \\
\hline & MET : NM_000245 & 15 & & & \\
\hline TP53 & NM_000546 & 8 & c. $811 \mathrm{G}>\mathrm{T}$ & p.Glu271* & $42.39 \%$ \\
\hline$A K T 1$ & NM_001014432 & 6 & c. $428 \mathrm{~A}>\mathrm{C}$ & p.His143Pro & $45.37 \%$ \\
\hline CCND1 & NM_053056 & 1 & c. $94 \mathrm{C}>\mathrm{G}$ & p.Leu32Val & $49.08 \%$ \\
\hline
\end{tabular}

* a nonsense mutation in a sequence of DNA that results in a premature stop codon.

TABLE 2 | Mutation profile of left lung tumor in the patient.

\begin{tabular}{|c|c|c|c|c|c|}
\hline Gene & Transcript & Exon & Nucleotide change & Alteration & Mutant allele frequency \\
\hline CCND1 & NM_053056 & 1 & c. $.94 \mathrm{C}>\mathrm{G}$ & p.L32V & $43.08 \%$ \\
\hline FL/1 & NM_002017 & 2 & c. $196 \mathrm{G}>\mathrm{A}$ & p.V66l & $44.52 \%$ \\
\hline ERBB2 & NM_004448 & 20 & c.2313_2324dup & p.772_775dup & $20.66 \%$ \\
\hline MED12 & NM_005120 & 36 & c. $4897 \mathrm{G}>\mathrm{T}$ & p.E1633* & $3.82 \%$ \\
\hline
\end{tabular}

* a nonsense mutation in a sequence of DNA that results in a premature stop codon.
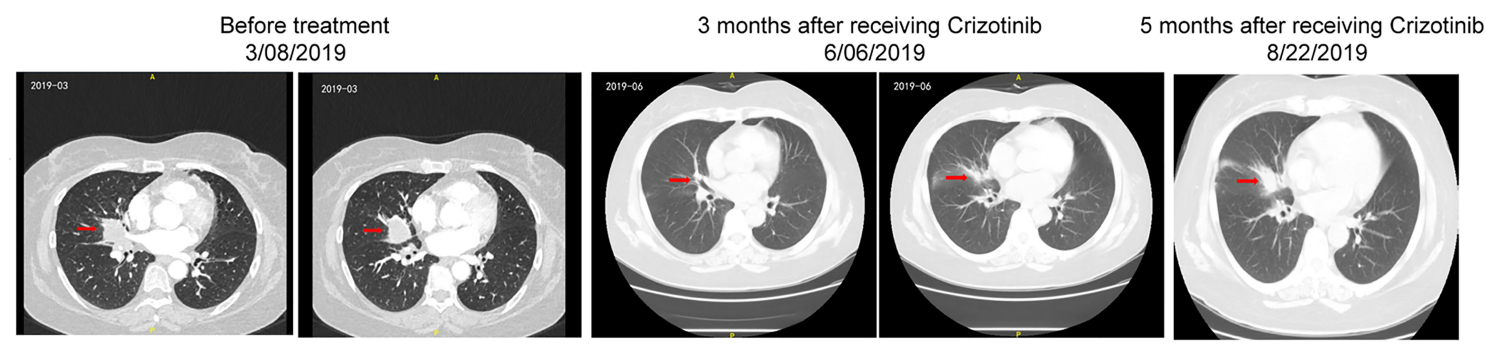

FIGURE 3 | Dynamic imaging of the soft tissue density nodule in the right middle lobe and near the hilum during the treatment with crizotinib.

TABLE 3 | Mutation profile of right lung tumor in the patient after resistance to crizotinib.

\begin{tabular}{|c|c|c|c|c|c|}
\hline Gene & Transcript & Exon & Nucleotide change & Alteration & Mutant allele frequency/copy number \\
\hline$A R I D 1 A$ & NM_006015 & 19 & c.5014del & p.V1672* & $2.3 \%$ \\
\hline$C D K N 2 A$ & NM_000077 & 1 & c.130del & p.Y44Tfs*9 & $1.3 \%$ \\
\hline ERBB2 & NM_004448 & 20 & c.2313_2324dup & p.Y772_A775dup & $31.1 \%$ \\
\hline PTPN13 & NM_080683 & 34 & c. $5564 \mathrm{G}>\mathrm{T}$ & p.R1855L & $12.7 \%$ \\
\hline ROBO1 & NM_002941 & 14 & c.1942del & p.1648Yfs*5 & $23.4 \%$ \\
\hline$S D H A$ & \multicolumn{4}{|c|}{ Gene amplification } & 2.6 \\
\hline
\end{tabular}

* a premature stop codon due to frameshift mutation or deletion mutation.

malignancy in the left lung, and no recurrence was observed. Meanwhile, she received crizotinib for unresectable malignancies in the right lung and obtained a 5-month PFS.

In recent years, the carcinogenic effect of driving genes has been increasingly understood due to the in-depth study of NSCLC. Therefore, the routine treatment of patients with NSCLC has drastically changed. MET fusion is a rare structural change compared with oncogenic mutations and represents an increasing pool of druggable targets in NSCLC. Furthermore, compared with mutations and amplification of MET, gene rearrangements are less documented, and the therapeutic relevance of more complex structural rearrangements remains largely unknown $(10,11)$. Translocated promoter region (TPR)$M E T$ fusion is one of the few known MET fusions. It is a rearrangement that fuses the oligomerization domain of $T P R$ with the completed tyrosine kinase domain of MET maintaining its oncogenicity (12).

Because the patient's sequencing results were negative for other oncogenic drivers, the ARL1-MET fusion may be the only potential druggable target. Crizotinib was the most appropriate choice for the patient, as it was initially developed as a $M E T$ inhibitor, and it has been proven to effectively inhibit $A L K$ and $M E T$ among several other kinases (8), showing good efficacy in preclinical and phase I studies (13). Plenker et al. reported two cases of genomic rearrangements, leading to gene fusion of KIF5B, STARD3NL, and MET. Both patients obtained a PR to crizotinib (1). Davies et al. reported a significant response to crizotinib in a patient with NSCLC positive for an HLA-DRB1- 


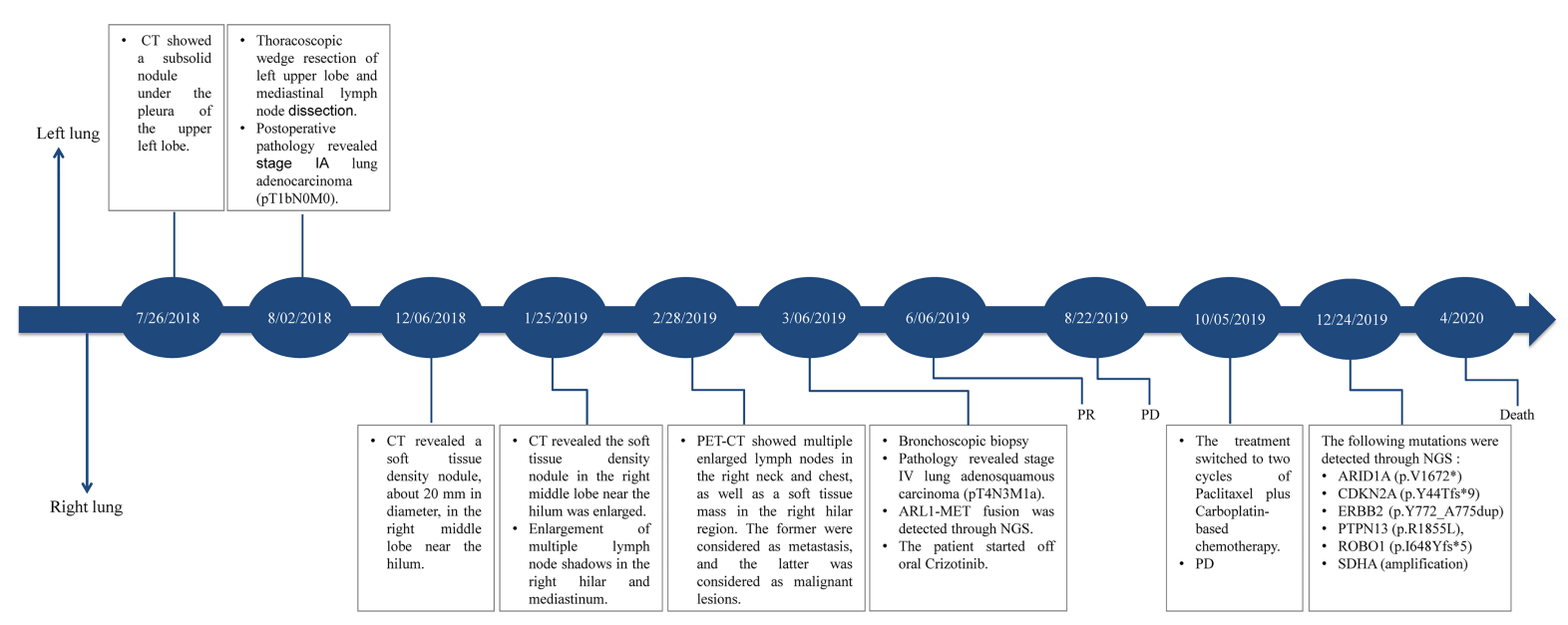

FIGURE 4 | Case timeline. * a premature stop codon due to frameshift mutation or deletion mutation.

MET fusion (2). Our results provide evidence that novel ARL1$M E T$ fusion may be an appealing target in patients with NSCLC.

MET tyrosine kinase inhibitors (MET-TKIs) are generally divided into three types depending on their structure and modes of binding with MET. Types I and II are ATP-competitive inhibitors. Type I inhibitors are further subdivided into types $\mathrm{Ia}$ and $\mathrm{Ib}$, depending on their interaction with the solvent front residue G1163. Type Ia inhibitors, such as crizotinib, interact with the solvent front residue G1163, whereas type Ib inhibitors, such as capmatinib, tepotinib, savolitinib, and APL-101, are independent of G1163 interaction. Type II MET-TKIs, such as cabozantinib, foretinib, merestinib, and glesatinib, are ATP competitors that bind to the inactive "DFG out" conformation of MET. Type III MET-TKIs, such as tivantinib, are non-ATPcompetitive allosteric inhibitors $(14,15)$. Among these METTKIs, crizotinib, capmatinib, and tepotinib are recommended by the Version 1, 2022 of the NCCN Guidelines for NSCLC for the patients with advanced NSCLC harboring MET exon 14 skipping mutations or high-level MET amplification. Also, capmatinib and tepotinib have been approved by the Food and Drug Administration (FDA) of the United States for the patients with metastatic NSCLC harboring MET exon 14 skipping mutations. Others are being evaluated in clinical trials.

Studies have identified two potential mechanisms underlying resistance to MET-TKIs. One was a secondary mutation of some active sites of $M E T$, like a point mutation at the tyrosine residue Y1230 of the MET tyrosine kinase domain and somatic M1268T mutation $(16,17)$. The other was activation of the downstream signaling pathways, including the RAS-MAPK, PI3K-AKT, and STATs pathways. NGS through the second right lung biopsy revealed that the original ARL1-MET fusion had "disappeared". Through pathology analysis, we speculate that the adenocarcinoma component harboring the ARL1-MET fusion had shrunk under treatment, leaving only the squamous component to grow. Thus, the novel ARL1-MET fusion did not disappear, but rather it was not present in the squamous tumor cells. This might explain the strikingly different mutation profiles in the tumors before and after treatment. However, ERBB2 insertion (p.Y772_A775dup) appeared which activates the bypass signaling pathway. This might be a mechanism underlying resistance in the patient (18).

There are a few limitations to our study. First, whether this finding means that all of the products of MET fusions could serve as oncogenic drivers in NSCLC remains uncertain. Second, it is still unclear why the patient rapidly developed resistance to crizotinib after 5 months of remission. Finally, there was only one case reported in our study, and more cases positive for ARL1-MET fusion are needed in the future to confirm the efficacy of crizotinib.

\section{CONCLUSION}

In conclusion, to the best of our knowledge, this study provides the first clinical evidence that the novel ARL1-MET fusion might be an actionable alteration in NSCLC. Further studies are needed to understand and overcome the potential mechanism(s) associated with resistance to MET inhibitors.

\section{DATA AVAILABILITY STATEMENT}

The original contributions presented in the study are included in the article/supplementary materials. Further inquiries can be directed to the corresponding author.

\section{ETHICS STATEMENT}

The studies involving human participants were reviewed and approved by the ethics committee of Tianjin Medical University General Hospital. The patients/participants provided their written informed consent to participate in this study. 


\section{AUTHOR CONTRIBUTIONS}

Conceptualization: DZ. Attending physicians for the patient: LK. Writing-original draft: QM. Editing draft: DZ. All authors read and approved the final manuscript.

\section{REFERENCES}

1. Plenker D, Bertrand M, de Langen AJ, Riedel R, Lorenz C, Scheel AH, et al. Structural Alterations of MET Trigger Response to MET Kinase Inhibition in Lung Adenocarcinoma Patients. Clin Cancer Res (2018) 24(6):1337-43. doi: 10.1158/1078-0432.CCR-17-3001

2. Davies KD, Ng TL, Estrada-Bernal A, Le AT, Ennever PR, Camidge DR, et al. Dramatic Response to Crizotinib in a Patient With Lung Cancer Positive for an HLA-DRB1-MET Gene Fusion. JCO Precis Oncol (2017) 2017(1): PO.17.00117. doi: 10.1200/PO.17.00117

3. Cho JH, Ku BM, Sun JM, Lee SH, Ahn JS, Park K, et al. KIF5B-MET Gene Rearrangement With Robust Antitumor Activity in Response to Crizotinib in Lung Adenocarcinoma. J Thorac Oncol (2018) 13(3):e29-31. doi: 10.1016/ j.jtho.2017.10.014

4. Zhu YC, Wang WX, Song ZB, Zhang QX, Xu CW, Chen G, et al. METUBE2H Fusion as a Novel Mechanism of Acquired EGFR Resistance in Lung Adenocarcinoma. J Thorac Oncol (2018) 13(10):e202-4. doi: 10.1016/ j.jtho.2018.05.009

5. Zhu YC, Wang WX, Xu CW, Zhang QX, Du KQ, Chen G, et al. Identification of a Novel Crizotinib-Sensitive MET-ATXN7L1 Gene Fusion Variant in Lung Adenocarcinoma by Next Generation Sequencing. Ann Oncol (2018) 29 (12):2392-3. doi: 10.1093/annonc/mdy455

6. Nelson AW, Schrock AB, Pavlick DC, Ali SM, Atkinson EC, Chachoua A. Novel SPECC1L-MET Fusion Detected in Circulating Tumor DNA in a Patient With Lung Adenocarcinoma Following Treatment With Erlotinib and Osimertinib. J Thorac Oncol (2019) 14(2):e27-9. doi: 10.1016/ j.jtho.2018.10.160

7. Rodig SJ, Shapiro GI. Crizotinib, a Small-Molecule Dual Inhibitor of the CMet and ALK Receptor Tyrosine Kinases. Curr Opin Investig Drugs (2010) 11 (12):1477-90.

8. Zou HY, Li Q, Lee JH, Arango ME, McDonnell SR, Yamazaki S, et al. An Orally Available Small-Molecule Inhibitor of C-Met, PF-2341066, Exhibits Cytoreductive Antitumor Efficacy Through Antiproliferative and Antiangiogenic Mechanisms. Cancer Res (2007) 67(9):4408-17. doi: 10.1158/0008-5472.CAN-06-4443

9. Yu CJ, Lee FJ. Multiple Activities of Arll GTPase in the Trans-Golgi Network. J Cell Sci (2017) 130(10):1691-9. doi: 10.1242/jcs.201319

10. Paik PK, Drilon A, Fan PD, Yu H, Rekhtman N, Ginsberg MS, et al. Response to MET Inhibitors in Patients With Stage IV Lung Adenocarcinomas Harboring MET Mutations Causing Exon 14 Skipping. Cancer Discov (2015) 5(8):842-9. doi: 10.1158/2159-8290.CD-14-1467

11. Seo JS, Ju YS, Lee WC, Shin JY, Lee JK, Bleazard T, et al. The Transcriptional Landscape and Mutational Profile of Lung Adenocarcinoma. Genome Res (2012) 22(11):2109-19. doi: 10.1101/gr.145144.112

\section{ACKNOWLEDGMENTS}

The authors wish to thank the patient and our colleagues in the Department of Pathology of Tianjin Medical University General Hospital for providing the pathological images.

12. Pal K, Bandyopadhyay A, Zhou XE, Xu Q, Marciano DP, Brunzelle JS, et al. Structural Basis of TPR-Mediated Oligomerization and Activation of Oncogenic Fusion Kinases. Structure (2017) 25(6):867-77.e3. doi: 10.1016/ j.str.2017.04.015

13. Cui JJ, Tran-Dube M, Shen H, Nambu M, Kung PP, Pairish M, et al. Structure Based Drug Design of Crizotinib (PF-02341066), a Potent and Selective Dual Inhibitor of Mesenchymal-Epithelial Transition Factor (C-MET) Kinase and Anaplastic Lymphoma Kinase (ALK). J Med Chem (2011) 54(18):6342-63. doi: $10.1021 / j m 2007613$

14. Gherardi E, Birchmeier W, Birchmeier C, Vande Woude G. Targeting MET in Cancer: Rationale and Progress. Nat Rev Cancer (2012) 12(2):89-103. doi: $10.1038 / \mathrm{nrc} 3205$

15. Cui JJ. Targeting Receptor Tyrosine Kinase MET in Cancer: Small Molecule Inhibitors and Clinical Progress. J Med Chem (2014) 57(11):4427-53. doi: $10.1021 / j m 401427 \mathrm{c}$

16. Qi J, McTigue MA, Rogers A, Lifshits E, Christensen JG, Janne PA, et al. Multiple Mutations and Bypass Mechanisms can Contribute to Development of Acquired Resistance to MET Inhibitors. Cancer Res (2011) 71(3):1081-91. doi: 10.1158/0008-5472.CAN-10-1623

17. Diamond JR, Salgia R, Varella-Garcia M, Kanteti R, LoRusso PM, Clark JW, et al. Initial Clinical Sensitivity and Acquired Resistance to MET Inhibition in MET-Mutated Papillary Renal Cell Carcinoma. J Clin Oncol (2013) 31(16): e254-8. doi: 10.1200/JCO.2012.46.4289

18. Luque-Cabal M, Garcia-Teijido P, Fernandez-Perez Y, Sanchez-Lorenzo L, Palacio-Vazquez I. Mechanisms Behind the Resistance to Trastuzumab in HER2-Amplified Breast Cancer and Strategies to Overcome it. Clin Med Insights Oncol (2016) 10(Suppl 1):21-30. doi: 10.4137/CMO.S34537

Conflict of Interest: The authors declare that the research was conducted in the absence of any commercial or financial relationships that could be construed as a potential conflict of interest.

Publisher's Note: All claims expressed in this article are solely those of the authors and do not necessarily represent those of their affiliated organizations, or those of the publisher, the editors and the reviewers. Any product that may be evaluated in this article, or claim that may be made by its manufacturer, is not guaranteed or endorsed by the publisher.

Copyright (c) $2022 \mathrm{Ma}$, Kong and Zhong. This is an open-access article distributed under the terms of the Creative Commons Attribution License (CC BY). The use, distribution or reproduction in other forums is permitted, provided the original author(s) and the copyright owner(s) are credited and that the original publication in this journal is cited, in accordance with accepted academic practice. No use, distribution or reproduction is permitted which does not comply with these terms. 